American Heart Association. Transgenic mice were generated and maintained at the University of Iowa Transgenic Animal Facility, which is supported in part by the College of Medicine and the Diabetes and Endocrinology Research Center. DNA sequencing was performed at the University of Iowa DNA Core Facility. Requests for materials should be addressed to Curt D. Sigmund at curt-sigmund@uiowa.edu. Address correspondence to Dr. Curt D. Sigmund, Chair, Molecular Biology Interdisciplinary Program, Director, Transgenic and Gene Targeting Facility Department of Internal Medicine and Physiology \& Biophysics, 2191 Medical Laboratory, The University of Iowa College of Medicine, Iowa City, IA 52242, USA.e-mail:curt-sigmund@uiowa.edu

Received 8 February 2001; accepted 9 May 2001.

David E. Stec, Satoshi Mori-
moto, and Curt D. Sigmund
The University of Iowa
College of Medicine
Iowa City, IA, USA

Iowa City, IA, USA

\section{AdEasy System Made Easier by Selecting the Viral Backbone Plasmid Preceding Homologous Recombination}

BioTechniques 31:260-262 (August 2001)

Recombinant adenovirus is widely used as a mammalian cell expression vector for multiple purposes including gene therapy (1) and noninvasive vaccination (5). It is urgent at this juncture to develop an efficient protocol for constructing adenovirus recombinants in a timely manner. The conventional approach to construct a replication-defective recombinant adenoviral vector requires a series of time-consuming and labor-intensive steps involving homologous recombination between two transfected plasmids in mammalian packaging cells (3). The finding that homologous recombination can be carried out in E. coli $(2,4)$ streamlined the procedure by allowing recombination to occur overnight in bacterial cells and obviated the need for plaque purification. The AdEasy system (4) is one of the fast-track systems for generating recombinant adenovirus by homologous recombination in E. coli. Typically, a linearized shuttle vector plasmid encoding kanamycin resistance is mixed with an adenoviral backbone plasmid (pAdEasy-1 or pAdEasy-2) encoding ampicillin resistance, followed by cotransformation into competent $E$. coli BJ5183 cells. Recombinants are subsequently selected for kanamycin resistance and identified by size in conjunction with restriction endonuclease analysis. Finally, recombinant adenoviral vectors are generated by transfecting the recombinant plasmid into a mammalian packaging cell line (e.g., 293 or 911 cells).

We report here that the key step in producing a recombinant plasmid in $E$. coli can be enhanced by pre-selecting the adenoviral backbone plasmid before the delivery of the shuttle vector plasmid. In this two-step transformation protocol, we electroporated an adenoviral backbone plasmid (i.e., pAdEasy-1) into E. coli BJ5183 cells, followed by growing transformants on LB agar plates containing $50 \mu \mathrm{g} / \mathrm{mL}$ ampicillin. A single colony containing pAdEasy-1 (BJ5183pAdEasy-1) was subsequently characterized by restriction analysis of the plasmid. BJ5183pAdEasy-1 cells were made electrocompetent as described (4) and kept in aliquots at $-80^{\circ} \mathrm{C}$. To trigger a recombination reaction, a shuttle vector plasmid encoding the Bacillus anthracis protective antigen (pShuttle-CMV. PA63) was digested with restriction endonuclease PmeI, and $25 \mathrm{ng}$ linearized plasmid DNA, without enzyme inactivation and purification, was directly electroporated into BJ5183pAdEasy-1 cells. Transformants were selected on LB agar plates containing $50 \mu \mathrm{g} / \mathrm{mL}$ kanamycin. Plasmid DNA was prepared from individual colonies using the QIAprep ${ }^{\circledR}$ spin miniprep kit (Qiagen, Valencia, CA, USA). The 9.2-kb pShuttle-CMV.PA63 plasmid and the 35-kb recombinant plasmid, both selectable by kanamycin resistance, could be clearly identified by size after fractionating supercoiled DNA in agarose gel.

As shown in Figure 1, transformation by mixing pShuttle-CMV.PA63 with pAdEasy-1 before electroporation as described (4) resulted in a success rate of $12 \%(2 / 17)$, whereas the new two-step transformation protocol generated recombinant plasmids at a frequency of $94 \%$ (16/17). The difference in outcomes between the two protocols was highly significant $(P<0.001$ by Fisher's exact test). BJ5183pAdEasy-1 competent cells that had been frozen at $-80^{\circ} \mathrm{C}$ for up to eight months were as efficient as their freshly prepared counterparts in generating recombinant plasmids. We envision that the enhanced selection of recombinants in antibiotics following two-step transformation may be attributed to productive recombination between incoming shuttle vector plasmids and pre-selected pAdEasy-1 plasmids that existed as autonomous replicons in E. coli cells. It is conceivable that only a small fraction of the pAdEasy-1 plasmid pool may be allowed to persist in E. coli cells following transformation because there is a high chance for a large plasmid [pAdEasy-1 is $33 \mathrm{~kb}$ in size (4)] to be defective (e.g., nicks along its long DNA strands), and/or the efficiency for 


\section{Benchmarks}

connecting a large plasmid to the cellular replication machinery may be low. Therefore, homologous recombination between a shuttle vector plasmid and an adenoviral backbone plasmid unable to exist as a replicon in $E$. coli cells is counterproductive for generating selectable recombinant plasmids because such recombinants are abortive. The two-step transformation protocol en- sures that homologous recombination occurs in a productive manner by elim inating defective and non-replicative adenoviral backbone plasmids in advance, thereby allowing a higher success rate during the selection for recombinants. Moreover, the step to inactivate PmeI with phenol/chloroform and purify the linearized shuttle vector plasmid as described (4) is no

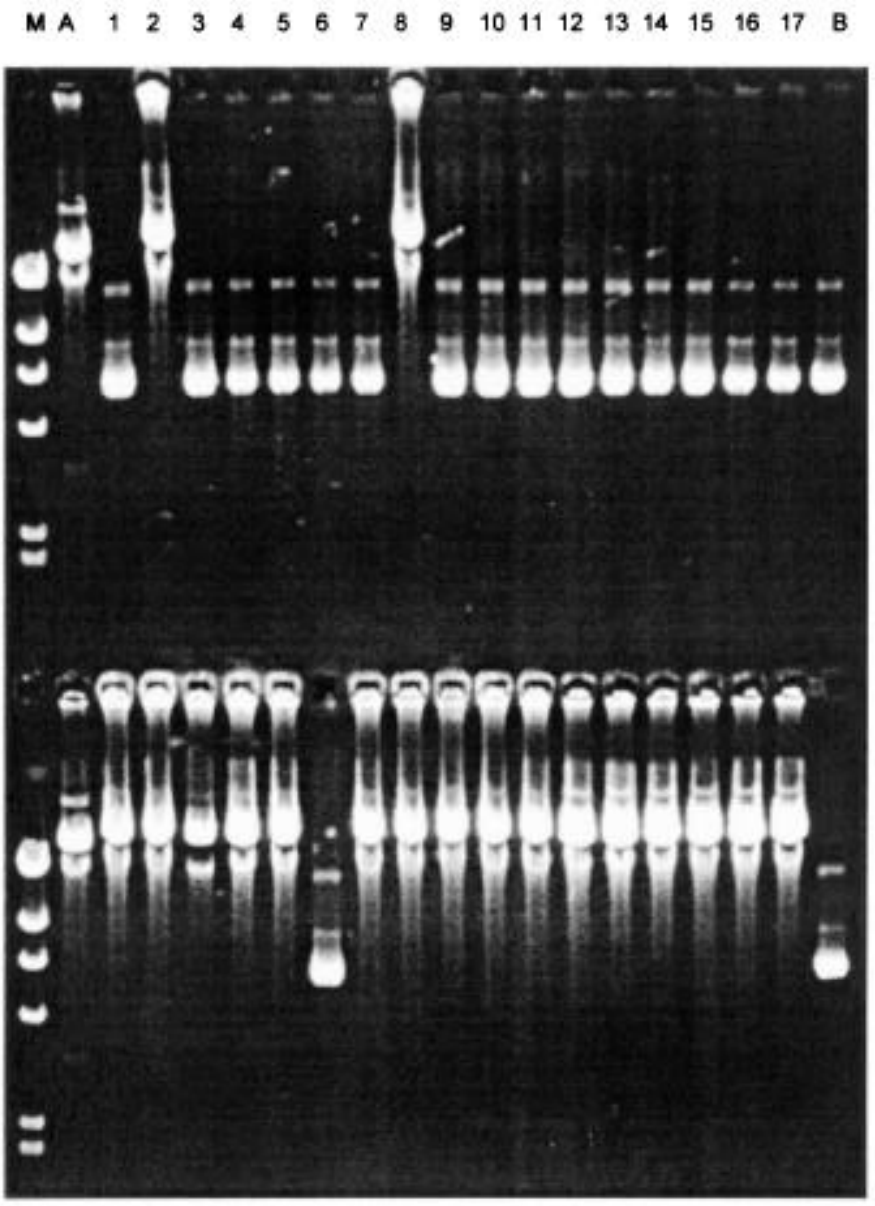

$\begin{array}{llllllllllllllllllll}\text { M A } & 18 & 19 & 20 & 21 & 22 & 23 & 24 & 25 & 26 & 27 & 28 & 29 & 30 & 31 & 32 & 33 & 34 & \text { B }\end{array}$

Figure 1. Enhanced generation of selectable plasmid recombinants following homologous recom bination in $\boldsymbol{E}$. coli cells by pre-selecting the adenoviral backbone plasmid. Homologous recombination between the pShuttle-CMV.PA63 plasmid $(9.2 \mathrm{~kb})$ and the pAdEasy-1 plasmid $(33 \mathrm{~kb})$ in E. coli BJ5183 cells was triggered by two different protocols: $(i)$ a one-step transformation protocol by which the two plasmids were mixed before electroporation as described (4) and (ii) a two-step transformation protocol that allows the linearized pShuttle-CMV.PA63 plasmid to be transformed into E. coli BJ5183pAdEasy-1 cells harboring the pAdEasy-1 plasmid as a pre-selected autonomous replicon. Lane $\mathrm{M}$, molecular weight marker (HindIII-cleaved $\lambda$ DNA) (Life Technologies, Rockville, MD, USA); lane A, supercoiled pAdEasy-1 DNA control; lane B, supercoiled pShuttle-CMV.PA63 DNA control; lanes 1-34, supercoiled plasmid DNA from individual kanamycin-resistant colonies following homologous recombination using either the one-step transformation protocol (lanes 1-17) or the two-step transformation protocol (lanes 18-34). DNA samples were fractionated in $0.8 \%$ agarose gel, stained with ethidium bromide, and visualized with the Gel-Doc ${ }^{\mathrm{TM}} 2000$ (Bio-Rad Laboratories, Hercules, CA, USA).

longer required because the need to mix the linearized shuttle vector plasmid with the supercoiled adenoviral backbone plasmid before transformation is negated. Overall, this two-step transformation protocol may have broad utility in systems that involve homologous recombination in bacteria.

\section{REFERENCES}

1.Benihoud, K., P. Yeh, and M. Perricaudet. 1999. Adenovirus vectors for gene delivery. Curr. Opin. Biotechnol. 10:440-447.

2.Chartier, C., E. Degryse, M. Gantzer, A. Dieterle, A. Pavirani, and M. Mehtali. 1996. Efficient generation of recombinant adenovirus vectors by homologous recombination in Escherichia coli. J. Virol. 70:4805-4810.

3.Graham, F.L. and L. Prevec. 1995. Methods for construction of adenovirus vectors. Mol. Biotechnol. 3:207-220.

4.He, T.C., S. Zhou, L.T. da Costa, J. Yu, K.W. Kinzler, and B. Vogelstein. 1998. A simplified system for generating recombinant adenoviruses. Proc. Natl. Acad. Sci. USA 95:2509-2514.

5.Tang, D.C., Z. Shi, and D.T. Curiel. 1997. Vaccination onto bare skin. Nature 388:729730 .

The AdEasy system was provided by Drs. Tong-Chuan He and Bert Vogelstein at the Johns Hopkins University. The Bacillus anthracis protective antigen gene was provided by Drs. Joanne McAndrew and Charles Turnbough, Jr. at the University of Alabama at Birmingham. Address correspondence to Dr. De-chu C. Tang, Department of Dermatology, University of Alabama at Birmingham, VH-501, 1670 University Blvd., Birmingham, AL 352940019, USA.e-mail: dctang@uab.edu

Received 13 March 2001; accepted 9 May 2001.

M. Zeng',2, S.K. Smith', F. Siegel ${ }^{1}$, Z. Shi ${ }^{1}$, K.R. Van Kampen $^{1}$, C.A. Elmets ${ }^{2}$, and D.C. Tang ${ }^{1,2}$

IVaxin, Inc.

${ }^{2}$ University of Alabama at Birmingham

Birmingham, AL, USA

For reprints of this or any other article, contact Reprints@BioTechniques.com 